\title{
Characterization of an immunogenic region of the Bm86 antigen as an improved vaccine against the Rhipicephalus microplus tick
}

\section{Caracterización de una región inmunogénica del antígeno Bm86 como vacuna mejorada contra la garrapata Rhipicephalus microplus}

Uriel Valdez-Espinoza ${ }^{1} \square$, Ninnet Gómez-Romero"回, Rubén Hernández-Ortiz'回, Edgar Castro-Saines'回, Rodolfo Lagunes-Quintanilla ${ }^{1}\left[{ }^{*}\right.$

${ }^{1}$ Centro Nacional de Investigación Disciplinaria en Salud Animal e Inocuidad, Instituto Nacional de Investigaciones Forestales, Agrícolas y Pecuarias. Carretera Federal Cuernavaca-Cuautla 8534, Col. Progreso, CP 62550. Jiutepec, Morelos, Mexico. ${ }^{2}$ Facultad de Medicina Veterinaria y Zootecnia, Universidad Nacional Autónoma de México. Avenida Universidad 3000, CP 04510. Mexico city, Mexico.

*Corresponding author

E-mail address: rodolfo.lagunes@gmail.com (R. Lagunes-Quintanilla).

Article history:

Received: 18 August 2021 / Received in revised form: 28 September 2021 / Accepted: / 22 October 2021 / Published online: 5 November 2021.

https://doi.org/10.29267/mxjb.2021.6.4.35

\begin{abstract}
One of the main health problems in cattle farming is infestations caused by ticks. The most important species is Rhipicephalus microplus, which affects $80 \%$ of bovine population worldwide. Its control is mainly based on the use of ixodicides. However, excessive and inappropriate use has generated multi-resistant strains of ticks. Alternatively, immune control has been proposed as an important method to reduce tick populations. The recombinant Bm86 vaccine is the only commercially available and reports variations in its efficacy due to polymorphisms in the gene's coding sequence. Therefore, this work aimed to characterize an immunogenic region of Bm86 strain $R$. microplus "Media Joya" to propose a vaccine candidate that is conserved and with minimal variability in its efficacy. Computational tools, molecular biology techniques, and phylogenetic analysis were used. As a result, an immunogenic region consisting of 178 amino acids and $98-100 \%$ identity/similarity were obtained concerning strains of $R$. microplus ticks from the Americas. Furthermore, is necessary to evaluate the
\end{abstract}


immunoprotective potential of the Bm86 region characterized in this work as an anti-tick vaccine.

Keywords: Bm86, R. microplus, ticks, vaccine.

\section{RESUMEN}

Uno de los principales problemas sanitarios de la ganadería bovina son las infestaciones ocasionadas por garrapatas. La especie más importante es Rhipicephalus microplus, la cual afecta al $80 \%$ de la población bovina del mundo. Su control se basa principalmente en el uso de ixodicidas, sin embargo, el uso excesivo e inadecuado ha generado cepas de garrapatas multiresistentes. Alternativamente, se ha propuesto al control inmunológico como un método importante para reducir las poblaciones de garrapatas, siendo la vacuna recombinante de Bm86 la única disponible comercialmente y con reportes de variaciones en su eficacia debido a la presencia de polimorfismos en el gen codificador. El objetivo de este trabajo fue caracterizar una región inmunogénica de la proteína Bm86 de $R$. microplus cepa "Media Joya" con el fin de proponer un candidato vacunal que sea conservado y con mínima variabilidad en cuanto a su eficacia. Se utilizaron herramientas computacionales, técnicas de biología molecular y análisis filogenéticos, y se obtuvo una región inmunogénica constituida por 178 aminoácidos y con $98-100 \%$ de identidad y similitud con respecto a cepas de garrapatas $R$. microplus provenientes del continente americano. Adicionalmente, se requiere evaluar el potencial inmunoprotector de la región de Bm86 caracterizada en este trabajo como vacuna anti-garrapatas.

Palabras clave: Bm86, garrapatas, R. microplus, vacuna.

\section{INTRODUCCIÓN}

Las garrapatas son ectoparásitos que ocasionan efectos negativos directos e indirectos en el ganado bovino (Pérez de León et al., 2020). Rhipicephalus microplus, conocida a nivel mundial como la garrapata común del ganado, tiene una amplia distribución dentro de las regiones tropicales y subtropicales de México (Estrada-Peña et al., 2006). Debido a su acción hematófaga, los efectos por infestaciones en los bovinos generan pérdidas económicas asociadas a la disminución en la producción pecuaria, así como también por su capacidad para transmitir patógenos intraeritrocíticos como Babesia bovis, $B$. bigemina y Anaplasma marginale, causantes de la Babesiosis y Anaplasmosis bovina; enfermedades que generan mortalidad en bovinos susceptibles (Rodríguez-Vivas et al., 2017).

El método de control más utilizado se basa en la aplicación de ixodicidas químicos, sin embargo, el uso continuo e inadecuado ha permitido el desarrollo de poblaciones de garrapatas resistentes a estos químicos, de tal forma que a la fecha existen en el país poblaciones con características de multiresistencia (Fernández-Salas et al., 2012; Rodríguez-Vivas et al., 2014), siendo una limitante para el control en los hatos bovinos de zonas geográficas donde se encuentra establecida. 
En México, el $70 \%$ del ganado bovino está expuesto a infestaciones por $R$. microplus según su distribución (Estrada-Peña et al., 2006). Datos actuales basados en modelos computacionales estiman un incremento en la distribución de $R$. microplus y las enfermedades que transmiten para los próximos años en América Latina (Marques et al., 2020). Esta información resalta la necesidad de mejorar los métodos de control contra $R$. microplus, y así proteger la sanidad de los hatos bovinos ante estas alteraciones siendo de suma importancia la implementación de programas integrales de control para la garrapata $R$. microplus en el ganado bovino que se encuentra distribuido en la región neotropical, y de esta manera fomentar un menor uso de ixodicidas, promoviendo la ganadería bovina sustentable (Rodríguez-Vivas et al., 2018). Con base en lo anterior, el control inmunológico es considerado como una de las alternativas más importantes a utilizar, debido a que su eficacia ha sido comprobada en diversos ensayos de campo (de la Fuente et al., 2007).

El uso de vacunas contra las garrapatas comenzó en la década de los 80's, sin embargo, fue hasta los años 90's en los que se logró comercializar la primera vacuna contra garrapatas R. microplus (Lagunes-Quintanilla \& Bautista-Garfias, 2020). Esta vacuna contiene una proteína denominada Bm86 la cual inicialmente fue identificada en las células del intestino medio de la garrapata $y$, posteriormente fue caracterizada inmuno-molecularmente, para ser expresada en un sistema eucarionte, formulando un inmunógeno con elevado grado de pureza (Canales et al., 1997). El mecanismo de acción del antígeno Bm86 se basa en la generación de anticuerpos contra esta proteína, logrando un efecto negativo en el desarrollo y en la capacidad reproductiva de las garrapatas que se alimentan de bovinos inmunizados (Lagunes-Quintanilla y Bautista-Garfias, 2020). La proteína Bm86 ha sido el único antígeno aprobado y distribuido comercialmente en México y otros países para su utilización en el control de $R$. microplus, el cual, mediante evaluaciones a nivel de campo demostró tener eficacia en diferentes cepas con antecedentes de resistencia a ixodicidas (Penichet et al., 1994; de la Fuente et al., 1999). Sin embargo, se han reportado polimorfismos genéticos en la secuencia de Bm86 entre diferentes cepas y aislados de $R$. microplus en el mundo. En cepas de distintas regiones de Sudamérica se han documentado variaciones que van desde el 3.4 - 6.8\% en la secuencia de aminoácidos (Sossai et al., 2005). Así mismo, en Tailandia se demostró que existe 91.5 - 93.5\% de identidad en la secuencia de aminoácidos de diferentes aislados con respecto al Bm86 que compone la vacuna comercial (Kaewmongkol et al., 2015). Adicionalmente, en México, se identificaron polimorfismos entre secuencias de Bm86 de diferentes aislados de $R$. microplus, provenientes de distintos estados del país (Martínez-Arzate et al., 2019).

Estas variaciones genéticas en el antígeno Bm86, podrían estar asociadas a una disminución en la eficacia de la vacunación en algunas regiones geográficas. Por tal motivo, el presente estudio tiene por objeto caracterizar una región inmunogénica de la proteína Bm86 de la garrapata $R$. microplus cepa "Media Joya" mediante algoritmos computacionales, con la finalidad de proponer un candidato a vacuna que sea conservado, con mínima variabilidad y alta eficacia cuando se utilice como método de control de garrapatas $R$. microplus. 


\section{MATERIALES Y MÉTODOS}

\subsection{Material biológico}

Se utilizó la cepa de garrapatas $R$. microplus "Media Joya", perteneciente al germoplasma del CENID-SAI, INIFAP, la cual se mantiene en condiciones controladas. Esta cepa fue aislada en el municipio de Tapalpa, Jalisco, México y se encuentra establecida en la Unidad de Artropodología desde el año 2001 como una cepa de referencia susceptible a ixodicidas.

\subsection{Análisis computacional de la proteína Bm86}

La base de datos del National Center for Biotechnology Information (NCBI) se utilizó para obtener la secuencia de aminoácidos de la proteína Bm86 (ADQ19690.1) con la finalidad de realizar un análisis computacional mediante diversos algoritmos bioinformáticos y seleccionar una región con características inmunogénicas y con mínima variabilidad genética. Se llevó a cabo un alineamiento múltiple con los programas CLUSTAL W y MUSCLE utilizando métodos progresivos e iterativos para comparar los resultados y obtener el mejor alineamiento. Para realizar los análisis de la estructura secundaria, se buscaron regiones hidrofóbicas utilizando la herramienta Protscale mediante el algoritmo Kyte \& Doolittle, regiones transmembranales con el programa TMHMM basado en modelos ocultos de Markov, presencia de péptido señal con SignalP 4.1, búsqueda de dominios funcionales con Pfam y SMART, y sitios de glicosilación con NetNGlyc 1.0. Dichos análisis permitieron verificar la presencia de regiones extracelulares, antigénicas y biológicamente funcionales para ser reconocidas por células de linaje inmunológico. La predicción de epítopos B se realizó con diferentes algoritmos, buscando características inmunogénicas como: Hidrofilicidad (Antigenic Plot), Antigenicidad (EMBOSS Antigenic) y epítopos lineales (Bepipred 2.0 y BCELL/IEDB Analysis Resource). Se seleccionaron epítopos lineales de al menos 15 aminoácidos de longitud con un índice de inmunidad de $0.5 \%$, con la finalidad de obtener cadenas cortas de aminoácidos en la región seleccionada, ya que, al no utilizar la proteína completa, la estructura nativa desaparece, así como los epítopos conformacionales que se localizan en la estructura terciaria y cuaternaria de la proteína. Una vez localizada la región de interés, se diseñaron un par de oligonucleótidos específicos mediante el programa OligoAnalyzer para su síntesis; Fw 5'CACCATGACCGAAGACTGTCGTGTGC -3' y Rv 5'- GAGCAGACTGTCGCAAAGG -3'. Finalmente, la representación esquemática de la región inmunogénica derivada de la proteína Bm86 se llevó a cabo mediante el programa Protter.

\subsection{Obtención de ácidos nucleicos y síntesis de ADN complementario}

Como material biológico se utilizaron $100 \mathrm{mg}$ de larvas de garrapatas $R$. microplus de 15 días de edad sin alimentar (cepa "Media Joya"), las cuales fueron pulverizadas en un mortero previamente congelado a $-80^{\circ} \mathrm{C}$. El aislamiento de $A R N$ se realizó por el método de Trizol $^{\circledR}$ (Thermo Fisher Scientific) con la finalidad de sintetizar ADN complementario (cDNA), mediante RT-PCR (Reacción en Cadena de la Polimerasa- 
Transcriptasa Reversa), el cual sirvió como plantilla en las reacciones de amplificación por PCR.

\subsection{Reacción en Cadena de la Polimerasa (PCR) y Clonación}

La reacción de PCR se llevó a cabo en tubos de microcentrífuga de pared delgada bajo el siguiente protocolo: 1X PCR buffer, $3 \mathrm{mM}$ de $\mathrm{MgCl}_{2}, 0.6 \mathrm{mM}$ de dNTPs, $0.5 \mathrm{U} / \mu \mathrm{l}$ de PfuUltra ADN polimerasa (Agilent $\left.{ }^{\circledR}\right), 2 \mu \mathrm{M}$ de oligonucleótidos y $100 \mathrm{ng}$ de cDNA, en un volumen final de $25 \mu$ l. Posteriormente, los amplicones obtenidos fueron separados por electroforesis en gel de agarosa al 1\% en buffer TBE (Tris, Borato, EDTA) para estimar el tamaño de los fragmentos amplificados por comparación con un marcador molecular de $1 \mathrm{~Kb}$ y visualizados por exposición UV en un transiluminador Gel Logic 1500 (Imaging System). La clonación de los productos de PCR se llevó a cabo utilizando el vector optimizado para expresión pET101/D-TOPO ${ }^{\circledR}$ (Invitrogen, Carlsbad, CA) realizando una reacción de ligación y la subsecuente transformación en células competentes TOP $10^{\circledR}$ de Escherichia coli. Se seleccionaron colonias recombinantes para realizar los ensayos de PCR de colonia y confirmar aquellas clonas que incorporaron el inserto. La extracción y purificación de plásmidos se llevó a cabo por el método de lisis alcalina y fueron secuenciados en la Unidad de Secuenciación del Instituto de Biotecnología de la Universidad Nacional Autónoma de México (IBt-UNAM).

\subsection{Análisis filogenético}

Se realizaron los análisis filogenéticos de la región seleccionada del gen $B m 86$ de la garrapata $R$. microplus (cepa "Media Joya"), con la finalidad de estudiar la relación genética que hay entre diversos aislados de la misma especie previamente reportados en la base de datos del NCBI. El árbol filogenético se construyó utilizando la paquetería de MEGA 7 (Kumar et al., 2016), para el cual se consideraron los siguientes parámetros: las distancias evolutivas se calcularon con base en el método Kimura de dos parámetros (Kimura, 1980) y como método de reconstrucción filogenética se utilizó el método de máxima verosimilitud (Guidon \& Gascuel, 2003). Adicionalmente, como soporte estadístico, se utilizaron 1000 réplicas de bootstraps (Felsestein, 1985). Finalmente, en el análisis se incluyeron 34 secuencias de garrapatas $R$. microplus y 2 secuencias que corresponden a garrapatas $R$. appendiculatus, las cuales fungieron como grupo externo.

\section{RESULTADOS}

Se caracterizó una región de la proteína Bm86 cepa "Media Joya" constituida por 178 aminoácidos, localizada entre los aminoácidos 266 y 442 de la proteína completa. En dicha región no se encontraron regiones transmembranales ni la presencia de péptido señal, por otra parte, se localizaron zonas hidrofílicas con valores por debajo de 1.6, lo cual indica de acuerdo a la composición fisicoquímica de cada residuo que no hay presencia de aminoácidos hidrofóbicos, presumiendo ser un fragmento completamente extracelular. Además, se localizaron dos sitios de $\mathrm{N}$-glicosilación situados en los residuos 348 y 382 con punto de corte de 0.5 y un dominio funcional de tipo Factor de 
Crecimiento Epidérmico (EGF). La predicción de regiones antigénicas y epítopos lineales de células $B$, sugiere que existen tres péptidos predichos como epítopos $B$, con un índice de inmunidad superior a $0.8 \%$ y una longitud entre 15 a 20 aminoácidos; indicativo de alta inmunogenicidad. En la Figura 1, se resumen las principales características de interés predichas en la región seleccionada de la proteína Bm86.

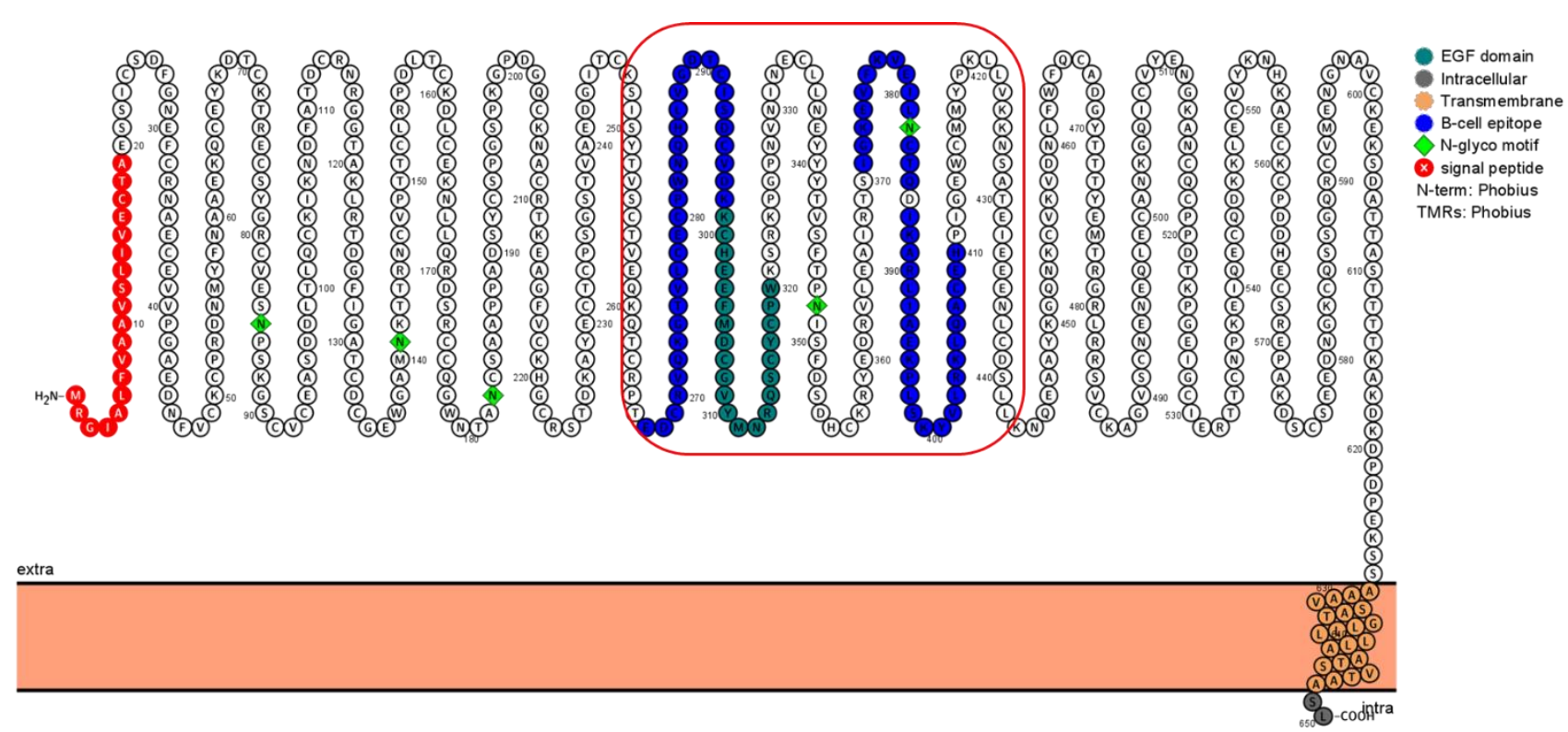

Fig. 1. Schematic representation of the region derived from the Bm86 protein. The amino acid sequence of the Bm86 protein is observed, and within the red box, the B epitopes (amino acids: 267-298, 371-385, 387-410) are seen in blue, the N-glycosylation sites (amino acids 348 and 382) in green and an EGF functional domain (amino acids 299-320) in turquoise. The transmembrane region is shown in pink, and the signal peptide is in red. The selected region appears to be an utterly extracellular fragment.

Fig. 1. Representación esquemática de la región derivada de la proteína Bm86. Se observa la secuencia de aminoácidos de la proteína Bm86 y dentro del recuadro en rojo se aprecian los epítopos B (aminoácidos: 267-298, 371-385, 387-410) en color azul, los sitios de $\mathrm{N}$-glicosilación (aminoácidos 348 y 382) en color verde y un dominio funcional EGF (aminoácidos 299-320) en color turquesa. En color rosa se observa la región transmembranal y en color rojo el péptido señal. La región seleccionada muestra ser un fragmento completamente extracelular.

Por otra parte, se realizó la amplificación por PCR de la región de interés del gen $B m 86$, obteniendo un amplicón de 534 pares de bases (Fig. 2A). Posteriormente, mediante ensayos de clonación se logró la obtención y purificación de 5 plásmidos recombinantes (Fig. 2B), los cuales fueron secuenciados en ambas direcciones con el fin de obtener una secuencia consenso. Al realizar el alineamiento múltiple de la secuencia derivada de la región de interés de la proteína Bm86 con las secuencias obtenidas de la base de datos del $\mathrm{NCBI}$, se obtuvo un $98-100 \%$ de identidad y similitud con respecto a las cepas provenientes del continente americano, sugiriendo un elevado grado de conservación entre cepas (Fig. 3); a diferencia de las cepas reportadas en África, Australia e India, 
donde se encontró un mayor número de sustituciones a nivel de aminoácidos; alcanzando $96-98 \%$ de identidad y similitud. Interesantemente, los péptidos predichos como epítopos B muestran un $99 \%$ de identidad en cepas provenientes del sur de Estados Unidos y de Sudamérica, en contraste con las originarias de África, Australia e India las cuales presentan $97 \%$.

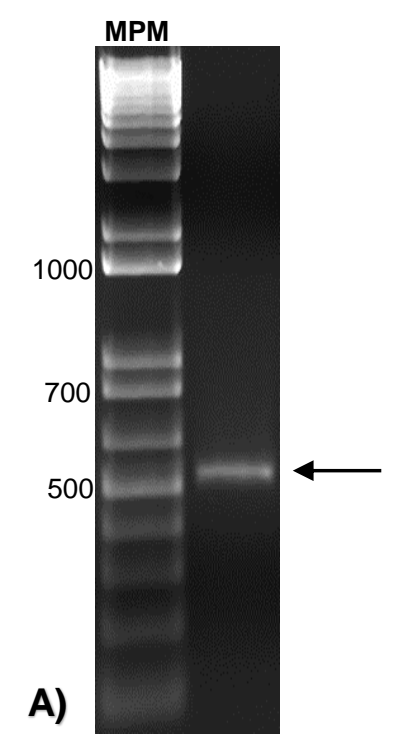

B)

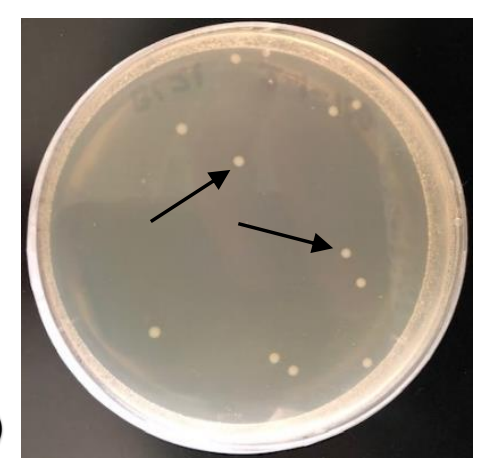

Fig. 2. Amplification and cloning of the selected region of Bm86. A) Product amplified by PCR corresponding to $534 \mathrm{bp}$. B) Visualization of recombinant $E$. coli colonies, including the selected region of Bm86. MPM: molecular weight marker (1 Kb). The black arrows indicate the amplified product and the recombinant colonies on LB-agar medium.

Fig. 2. Amplificación y clonación de la región seleccionada de Bm86. A) Producto amplificado por PCR equivalente a 534 bp. B) Visualización de colonias recombinantes de $E$. coli que incorporaron la región seleccionada de Bm86. MPM: marcador de peso molecular $(1 \mathrm{~Kb})$. Las flechas en color negro indican el producto amplificado y las colonias recombinantes en medio LB-agar.

Con respecto a la reconstrucción filogenética del fragmento de 534 nucleótidos derivados del gen Bm86 cepa "Media Joya", muestra la formación de dos clados definidos representados por las especies $R$. microplus y $R$. appendiculatus, en donde se observa que la secuencia caracterizada en este estudio se agrupa en el clado que pertenece a la especie $R$. microplus (Fig. 4). El análisis filogenético describe que la cepa "Media Joya" presenta una mayor similitud con aislados provenientes de regiones del sur de Estados Unidos y Sudamérica, en comparación con secuencias de aislados originarios de África, Australia e India. Con base en la relación filogenética y el análisis comparativo entre secuencias, es posible inferir que la región caracterizada genéticamente pertenece a una región conservada que se comparte en aislados de las diversas regiones geográficas reportadas en el continente americano. 


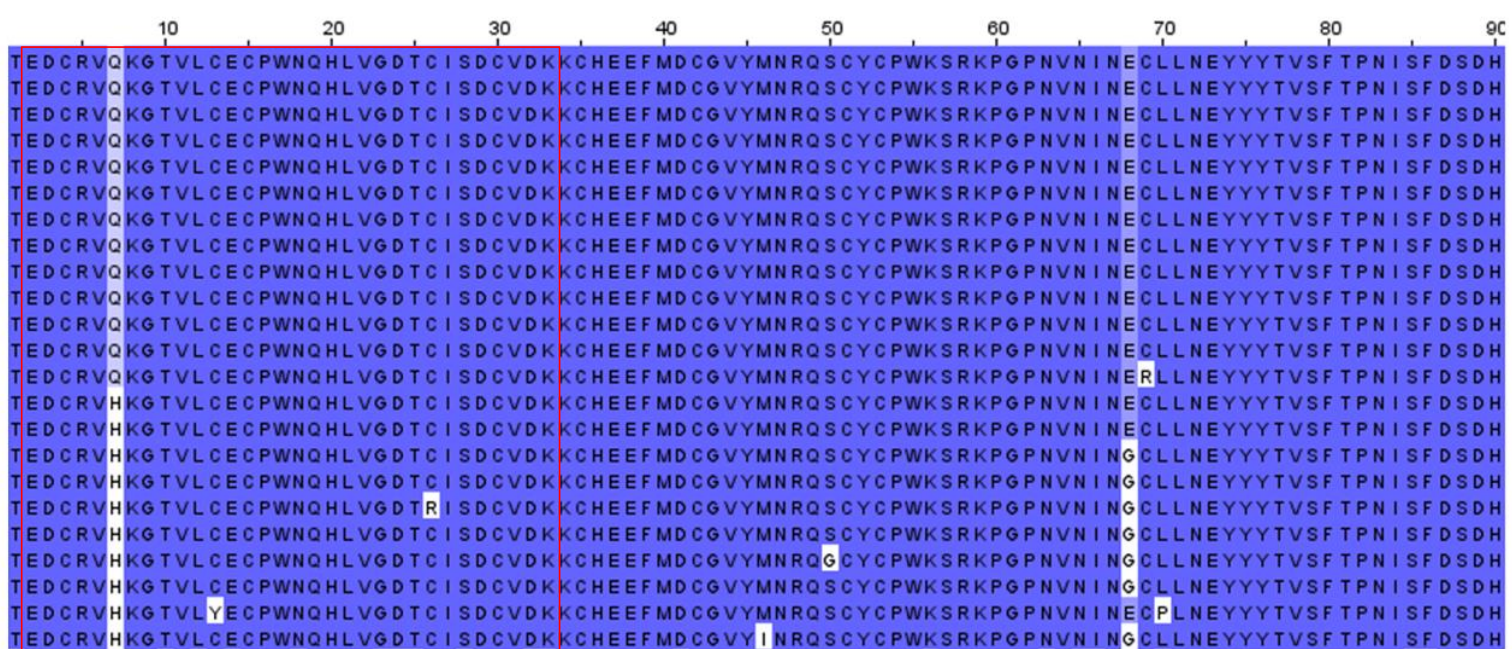

\section{R_microplus_cepa_Media}

HQ014398.1R microplus_aislado_Zapata 11 HQ014396.1_R_microplus_aislado_Starr 1 USA HQ014394.1_R_microplus_aislado_Zapata_12 USA HQ014393.1_R_microplus_aislado_Zapata_1_USA HQ014391.1_R_microplus_aislado_Zapata_5_USA HQ0 14388.1_R_microplus_aislado_Start_5_USA HQ014385.1_R_microplus_aislado_Starr_3_USA FJ456928.1_R_microplus_strain_Susceptible_Mexico HQ014397.1_R_microplus_aislado_Starn_2_USA HQ014392.1_R_microplus_aislado_Zapata_10_USA EU352677.1_R_microplus_aislado_Campo_Grande_Brasil AF 150891.2_B_microplus_Bm95_Argentina M29321.1_B_microplus_BMB6_Australia XM_037422513.1_R_microplus_LOC119171703 MG002404.1_R_microplus_aislado_ST_Australia MG002403.1_R_microplus_aislado_SZPLG_Australia MG002402.1_R_microplus_aisaldo_WC_Australia MG002401_R - microplus_Mozambique_Africa EU191620._R_microplus_cepa_Asozambique_Afica MG002399 - R microplus_cepa_Mozambique_Aatica HQ166286.1_R_microplus_BM_india

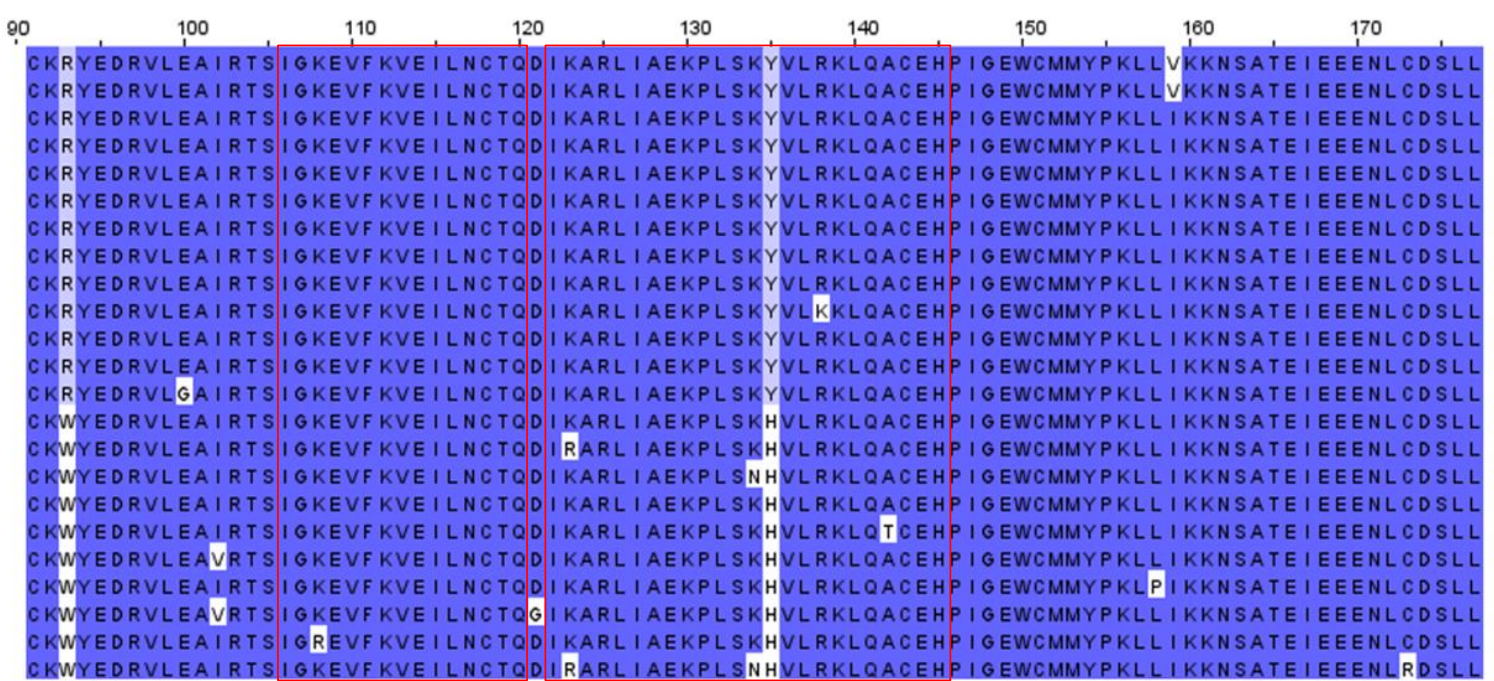

Fig. 3. Multiple alignment of the amino acids sequence derived from the protein Bm86 strain "Media Joya" of $R$. microplus. Conserved amino acids are shown in blue and variables in gray and white. Boxes in red indicate peptides predicted as B epitopes.

Fig. 3. Alineamiento múltiple de la secuencia de aminoácidos derivada de la proteína Bm86 cepa "Media Joya" de $R$. microplus. Los aminoácidos conservados se muestran en color azul y los variables en color gris y blanco. Los cuadros en color rojo indican los péptidos predichos como epítopos B. 


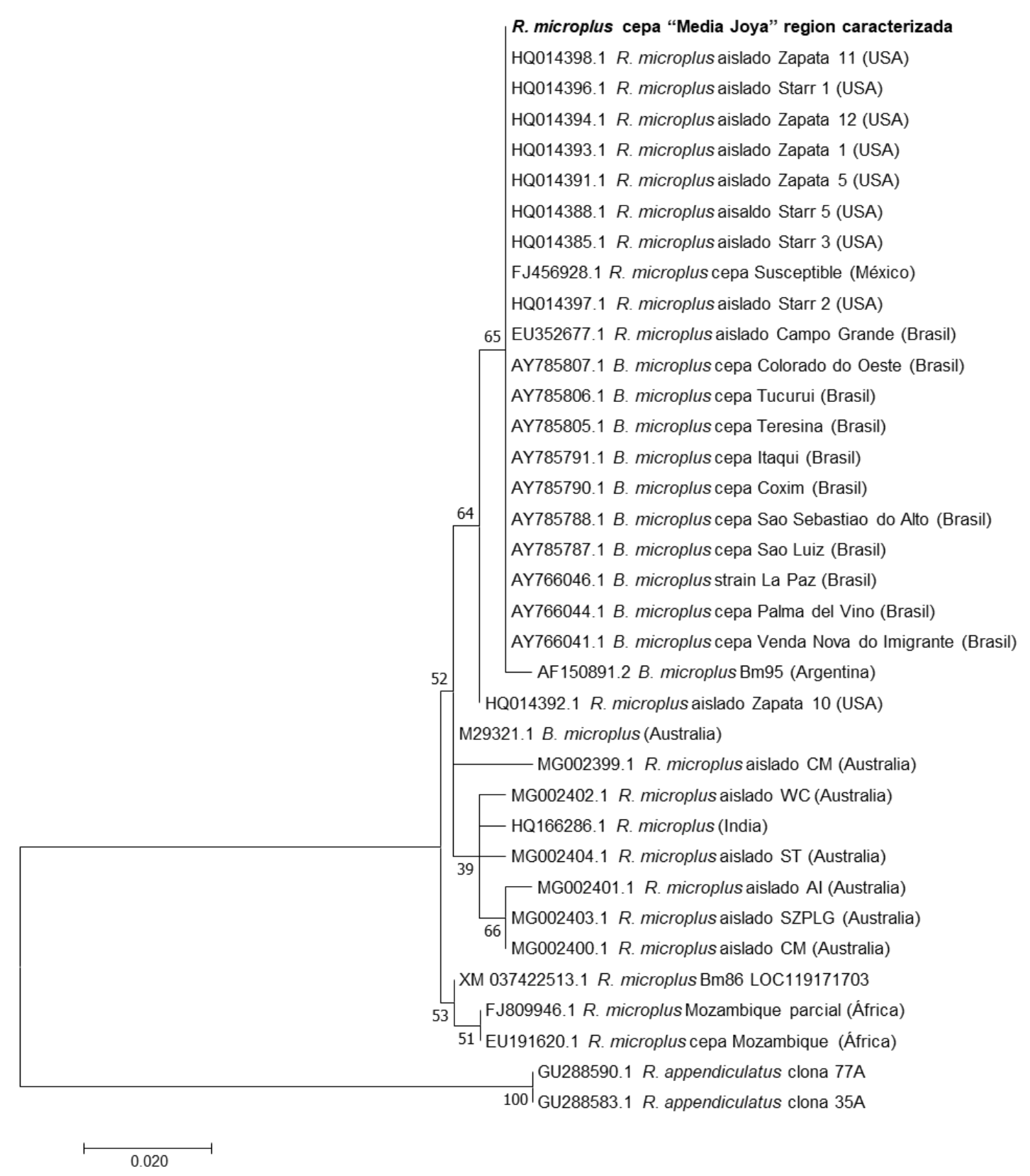

Fig. 4. Phylogenetic analysis of the characterized region of Bm86. The phylogenetic tree was constructed by the maximum likelihood method using the characterized sequence of Bm86 ("Media Joya") and the strains and isolates of ticks $R$. microplus previously reported in the NCBI. The numbers in the nodes indicate bootstrap values and posterior probability of the Bayesian analysis. Each sequence is identified with its origin and accession number.

Fig. 4. Análisis filogenético de la región caracterizada de Bm86. El árbol filogenético fue construido por el método de máxima verosimilitud utilizando la secuencia caracterizada de Bm86 ("Media Joya") y las cepas y aislados de garrapatas $R$. microplus previamente reportados en el NCBI. Los números en los nodos indican valores de bootstrap y 
probabilidad posterior del análisis Bayesiano. Cada secuencia se identifica con su origen y número de acceso.

\section{DISCUSIÓN}

El antígeno Bm86 continúa siendo la única vacuna disponible en la industria farmacéutica veterinaria para el control inmunológico de garrapatas $R$. microplus en el ganado bovino (Almazán et al., 2018). Sin embargo, existen reportes que indican la presencia de polimorfismos genéticos, los cuales están involucrados en la variabilidad de la eficacia en la vacunación de diversas regiones geográficas del mundo (Casquero et al., 2012).

El diseño de antígenos vacunales mediante análisis bioinformáticos utilizando regiones conservadas entre diversos aislados y/o cepas, se ha planteado como una estrategia de utilidad para el desarrollo de vacunas contra diversos patógenos (Mosqueda et al., 2012). El uso de antígenos conservados para mejorar la eficacia de las vacunas contra garrapatas es de especial importancia cuando se trata de vacunas basadas en la secuencia de un solo gen. Esto puede ser un modelo a emplear en la caracterización de regiones específicas que estimulen una respuesta inmune más eficaz contra las garrapatas $R$. microplus. Los resultados obtenidos en el presente estudio, muestran que la región seleccionada de Bm86 presenta características de interés que podrían mejorar la eficacia como vacuna; tres péptidos predichos como posibles epítopos de células B, dos sitios de $\mathrm{N}$-glicosilación y ser completamente extracelular. Las características inmunogénicas encontradas, podrían significar un incremento en la activación de las células del sistema inmune debido a que las glicosilaciones de tipo $\mathrm{N}$ - y O-glicosilación están relacionadas con la hiperantigenicidad (Daly \& Hearn, 2005), la estabilidad, el plegamiento y la actividad biología de las proteínas (Lis \& Sharon, 1993), así como se ha reportado en proteínas de membrana del virus de la influenza (Hütter et al., 2013). Con respecto a la estructura secundaria, las regiones predichas concuerdan con resultados previos (Rand et al., 1989; Tellam et al., 1992) donde se reconoce la ausencia de regiones transmembranales y presencia de un péptido señal en el extremo $\mathrm{N}$-terminal. Adicionalmente, esta región presentó un $98-100 \%$ de identidad y similitud entre las secuencias de Bm86 analizadas del continente americano, sugiriendo un alto grado de conservación. Estos datos son de gran relevancia ya que se ha reportado que una diferencia mayor al $2.8 \%$ en la secuencia de aminoácidos en Bm86 es suficiente para causar variación en la eficacia de la vacunación contra $R$. microplus. Este fenómeno ha sido descrito previamente en las secuencias genéticas de Bm86 entre aislados de México, Cuba y Argentina (García-García et al., 1999). De acuerdo a los resultados obtenidos, posiblemente la región caracterizada en esta investigación podría generar una respuesta inmune eficaz debido al elevado grado de conservación que presenta, sugiriendo ser un candidato potencial si se utiliza como vacuna contra garrapatas $R$. microplus en diferentes regiones geográficas.

Recientemente, se han realizado trabajos utilizando genes de vital importancia para Babesia spp., demostrando que la selección bioinformática de regiones hidrofílicas, inmunogénicas y conservadas permiten identificar péptidos con elevado potencial para el desarrollo de vacunas (Hernández-Silva et al., 2018; Mosqueda et al., 2019; Barreda et al., 2020). Por otro lado, existen investigaciones realizadas previamente donde se ha 
evidenciado que la inmunización de péptidos derivados del antígeno Bm86 son capaces de generar una respuesta inmune protectora, que se refleja en un mejor reconocimiento por los anticuerpos anti-Bm86 en el sitio blanco (intestino medio) de la garrapata $R$. microplus (Patarroyo et al., 2002, 2009). Sin embargo, en dicho estudio se analizó la capacidad antigénica de los péptidos utilizando únicamente un algoritmo para su elección. Si bien, los péptidos predichos poseen cualidades inmunogénicas, la elevada concentración y la dosificación poco práctica para su aplicación representa una desventaja a considerar en la formulación de vacunas para bovinos.

En el presente trabajo, uno de los péptidos predichos como epítopo B coincide con uno de los evaluados por Patarroyo et al., (2002). En contraste, el análisis computacional se realizó utilizando diferentes algoritmos para predecir sitios de hidrofilicidad, antigenicidad y de epítopo $\mathrm{B}$ lineal, lo cual permitió determinar que la región seleccionada posee características inmunogénicas relevantes en comparación con los péptidos reportados previamente. Adicionalmente, la región seleccionada presenta una longitud de 178 aminoácidos y contiene tres epítopos B líneales, evidenciando diferencias en peso molecular en comparación con los péptidos evaluados anteriormente (Patarroyo et al., 2002). En este aspecto, al evaluar el potencial inmunoprotector de la región de Bm86 caracterizada en este estudio podría generar una elevada respuesta inmunológica (Pavón et al., 2016) a la dosis y concentración que convencionalmente se utilizan en bovinos contra garrapatas $R$. microplus (MendozaMartínez et al., 2021).

Finalmente, las herramientas bioinformáticas representan una alternativa de suma utilidad para diseñar y proponer antígenos candidatos para el desarrollo de vacunas contra garrapatas y otros patógenos. En este estudio, se caracterizó una región inmunogénica derivada de la proteína Bm86 de la cepa de garrapatas $R$. microplus "Media Joya", la cual podría ser utilizada como una propuesta para candidato vacunal debido a las propiedades inmunogénicas y de mínima variabilidad encontradas. El uso de vacunas como parte de un control integral vislumbra ser un método efectivo contra garrapatas $R$. microplus que permitirá mejorar la salud animal, disminuir la contaminación ambiental y reducir el uso intensivo de ixodicidas.

\section{AGRADECIMIENTOS}

Proyecto parcialmente financiado con fondos fiscales INIFAP, N SIGI: 10533234452 , otorgado al Dr. Rodolfo E. Lagunes Quintanilla para la realización de los experimentos correspondientes.

\section{CONFLICTO DE INTERESES}

Los autores declaran que no existe conflicto de intereses.

\section{REFERENCIAS}

Almazán C., Tipacamu G. A., Rodríguez S., Mosqueda J. \& Pérez de León A. 2018. Immunological control of ticks and tick-borne diseases that impact cattle health and production. Frontiers in Bioscience. 1(23): 1535-1551. https://doi.org/10.2741/4659 
Barreda D., Hidalgo-Ruiz M., Hernandez-Ortiz R., Ramos J. A., Galindo-Velasco E. \& Mosqueda J. 2020. Identification of conserved peptides containing B-cell epitopes of Babesia bovis AMA-1 and their potential as diagnostics candidates. Transboundary and Emerging Diseases. 2: 60-68. https://doi.org/10.1111/tbed.13213

Canales M., Enríquez A., Ramos E., Cabrera D., Dandie H., Soto A., Falcón V., Rodríguez M. \& de la Fuente J. 1997. Large-scale production in Pichia pastoris of the recombinant vaccine Gavac against cattle tick. Vaccine. 5(4): 414-22. https://doi.org/10.1016/S0264-410X(96)00192-2

Casquero C. R., Pérez de León A. A., Pereira L. L., da Silva P. L., Gonçalves J. A. \& Andreotti R. 2012. Bovine immunoprotection against Rhipicephalus (Boophilus) microplus with recombinant Bm86-Campo Grande antigen. Revista Brasileira de Parasitologia Veterinária. 21(3): 254-262. https://doi.org/10.1590/s1984-29612012000300014

Daly R. \& Hearn M. 2005. Expression of heterologous proteins in Pichia pastoris: a useful experimental tool in protein engineering and production. Journal Of Molecular Recognition.18: 119-138. https://doi.org/10.1002/jmr.687

de la Fuente J., Almazán C., Canales M., Pérez de la Lastra J. M., Kocan K. M. \& Willadsen P. 2007. A ten-year review of commercial vaccine performance for control of tick infestations on cattle. Animal Health Research Reviews. 8(1): 23-28. https://doi.org/10.1017/S1466252307001193

de la Fuente J., Rodríguez M., Montero C., Redondo M., García-García J. C., Méndez L., Serrano E., Valdés M., Enríquez A., Canales M., Ramos E., Boué O., Machado H. \& Lleonart R. 1999. Vaccination against ticks (Boophilus spp.): the experience with the Bm86-based vaccine Gavac. Genetic analysis: biomolecular engineering. 15(3-5): 143148. https://doi.org/10.1016/S1050-3862(99)00018-2

Estrada-Peña A., García Z. \& Sánchez H. F. 2006. The distribution and ecological preferences of Boophilus microplus (Acari: Ixodidae) in Mexico. Experimental and Applied Acarology. 38(4): 307-316. https://doi.org/10.1007/s10493-006-7251-2

Felsenstein J. 1985. Confidence limits on phylogenies: an approach using the bootstrap. Evolution. 39:783-791. https://doi.org/10.2307/2408678

Fernandez-Salas A., Rodríguez-Vivas R. I. \& Alonso-Díaz M. A. 2012. First report of a Rhipicephalus microplus tick population multi-resistant to acaricides and ivermectin in the Mexican tropics. Veterinary Parasitology. 183(3-4): 338-342. https://doi.org/10.1016/j.vetpar.2011.07.028

García-García J. C., Gonzalez I. L., González D. M., Valdés M., Méndez L., Lamberti J., D'Agostino B., Citroni D., Fragoso H., Ortiz M., Rodríguez M. \& de la Fuente J. 1999. Sequence variations in the Boophilus microplus Bm86 locus and implications for 
immunoprotection in cattle vaccinated with this antigen. Experimental and Applied Acarology. 23(11): 883-895. https://doi.org/10.1023/a:1006270615158.

Guidon S. \& Gascuel O. 2003. A simple, fast and accurate algorithm to estimate large phylogenies by Maximum Likelihood. Systematic Biology. 52: 696-704. https://doi.org/10.1080/10635150390235520

Hernández-Silva D. J., Valdez-Espinoza U. M., Mercado-Uriostegui M. A., AguilarTipacamú G., Ramos-Aragón J. A., Hernández-Ortiz R., Ueti M. \& Mosqueda J. 2018. Immunomolecular characterization of MIC-1, a novel antigen in Babesia bigemina, which contains conserved and immunodominant B-Cell epitopes that induce neutralizing antibodies. Veterinary Sciences. 5(2): 32. https://doi.org/10.3390/vetsci5020032

Hütter J., Rödig J., Höper D., Seeberger P., Reichl U., Rapp E. \& Lepenies B. 2013. Toward animal cell culture-based influenza vaccine design: viral hemagglutinin $\mathrm{N}$ glycosylation markedly impacts immunogenicity. The Journal of Inmunology.190: 220230. https://doi.org/10.4049/jimmunol.1201060

Kaewmongkol S., Kaewmongkol G., Inthong N., Lakkitjaroen N., Sirinarumitr T., Berry C. M., Jonsson N. N., Stich R. W. \& Jittapalapong S. 2015. Variation among Bm86 sequences in Rhipicephalus (Boophilus) microplus ticks collected from cattle across Thailand. Experimental and Applied Acarology. 66(2): 247-256. https://doi.org/10.1007/s10493-015-9897-0.

Kimura M. 1980. A simple method for estimating evolutionary rates of base substitutions through comparative studies of nucleotide sequences. Journal of Molecular Evolution. 16: 111-120. https://doi.org/10.1007/bf01731581

Kumar S., Stecher G. \& Tamura K. 2016. MEGA 7: Molecular Evolutionary Genetics Analysis versipm 7.0 for bigger datasets. Molecular Biology and Evolution. 33: 18701874. https://doi.org/10.1093/molbev/msw054

Lagunes Q. R. E. \& Bautista-Garfias C. R. 2019. El control inmunológico: Una alternativa contra garrapatas del ganado bovino. Ecosistemas $Y$ Recursos Agropecuarios. 7(1). https://doi.org/10.19136/era.a7n1.2263

Lis H. \& Sharon N. 1993. Protein glycosylation. Structural and functional aspects. Europan Journal of Biochemistry. 218: 1-27. https://doi.org/10.1111/j.14321033.1993.tb18347.x.

Marques R., Krüger R. F., Peterson A. T., de Melo L. F., Vicenzi N. \& Jiménez-García D. 2020. Climate change implications for the distribution of the babesiosis and anaplasmosis tick vector, Rhipicephalus (Boophilus) microplus. Veterinary Research. 51(1): 81. https://doi.org/10.1186/s13567-020-00802-z. 
Martínez-Arzate S. G., Sánchez-Bermúdez J. C., Sotelo-Gómez S., Diaz-Albiter H. M., Hegazy-Hassan W., Tenorio-Borroto E., Barbabosa-Pliego A. \& Vázquez-Chagoyán J. C. 2019. Genetic diversity of Bm86 sequences in Rhipicephalus (Boophilus) microplus ticks from Mexico: analysis of haplotype distribution patterns. BMC Genetics. 20(1): 56. https://doi.org/10.1186/s12863-019-0754-8

Mendoza-Martínez N., Alonso-Díaz M. A., Merino O., Fernández-Salas A. \& LagunesQuintanilla R. 2021. Protective efficacy of the peptide Subolesin antigen against the cattle tick Rhipicephalus microplus under natural infestation. Veterinary Parasitology. 299: 109577. https://doi.org/10.1016/j.vetpar.2021.109577

Mosqueda G. J., Falcón N. A., Ramos A. J., Canto A. G. \& Camacho-Nuez M. 2012. Estrategias genómicas y moleculares para el control de la babesiosis bovina. Revista Mexicana de Ciencias Pecuarias. 3(1): 51-59.

Mosqueda J., Hidalgo-Ruiz M., Calvo-Olvera D. A., Hernandez-Silva D. J., Ueti M. W., Mercado-Uriostegui M. A., Rodriguez A., Ramos-Aragon J. A., Hernandez-Ortiz R., Kawazu S. I. \& Igarashi I. 2019. RON2, a novel gene in Babesia bigemina, contains conserved, immunodominant B-cell epitopes that induce antibodies that block merozoite invasion. Parasitology. 146(13):

1646-1654. https://doi.org/10.1017/S0031182019001161

Patarroyo J. H., Portela R. W., De Castro R. O., Pimentel J. C., Guzman F., Patarroyo M. E., Vargas M. I., Prates A. A. \& Mendes M. A. 2002. Immunization of cattle with synthetic peptides derived from the Boophilus microplus gut protein (Bm86). Veterinary Immunolgy and Immunopathology. 88(3-4): 163-72. https://doi.org/10.1016/s0165$\underline{2427(02) 00154-x}$

Patarroyo J. H., Vargas M. I., González C. Z., Guzman F., Mar-tins-Filho O. A., Afonso L. C. C., Valente F. L., Peconick A. P., Marciano A. P., Patarroyo A. M. \& Sossai S. 2009. Immune response of bovines stimulated by synthetic vaccine $\mathrm{SBm} 7462^{\circledR}$ against Rhipicephalus (Boophilus) microplus. Veterinary Parasitology. 166: 333-339. https://doi.org/10.1016/j.vetpar.2009.09.036

Pavón R. L., Jiménez M. M. C. \& Garcés A. M. E. 2016. Inmunología molecular, celular y traslacional. Editorial Wolters Kluwer. Barcelona. pp:152-155.

Penichet M., Rodriguez M., Castellano O., Mandado S., Rojas Y., Rubiera R., Sanchez P., Lleonart R. \& de la Fuente J. 1994. Detection of Bm86 antigen in different strains of Boophilus microplus and effectiveness of immunization with recombinant Bm86. Parasite Immunology. 16(9): 493-500. https://doi.org/10.1111/j.13653024.1994.tb00377.x

Pérez de León A. A., Mitchell R. D. \& Watson D. W. 2020. Ectoparasites of Cattle. The Veterinary clinics of North America. Food Animal Practice. 36(1): 173-185. https://doi.org/10.1016/j.cvfa.2019.12.004 
Rand K. N., Moore T., Sriskantha A., Spring K., Tellam R., Willadsen P. \& Cobon G. S. 1989. Cloning and expression of a protective antigen from the cattle tick Boophilus microplus. Proceedings of the National Academy of Sciences of the United States of America. 86(24): 9657-9661. https://doi.org/10.1073/pnas.86.24.9657

Rodríguez-Vivas R. I., Grisi L., Pérez de León A. A., Silva V. H., Torres-Acosta J. F., Fragoso S. H., Romero S. D., Rosario C. R., Saldierna F. \& García C. D. 2017. Evaluación del impacto económico potencial de los parásitos del ganado bovino en México. Revista Mexicana de Ciencias Pecuarias. 8(1): 61-74. https://doi.org/10.22319/rmcp.v8i1.4305

Rodríguez-Vivas R. I., Jonsson N. N. \& Bhushan C. 2018. Strategies for the control of Rhipicephalus microplus ticks in a world of conventional acaricide and macrocyclic lactone resistance. Parasitology Research. 117(1): 3-29. https://doi.org/10.1007/s00436-017-5677-6

Rodríguez-Vivas R. I., Pérez-Cogollo L. C., Rosado-Aguilar J. A., Ojeda-Chi M. M., Trinidad-Martinez I., Miller R. J., Li A. Y., de León A. P., Guerrero F. \& Klafke G. 2014. Rhipicephalus (Boophilus) microplus resistant to acaricides and ivermectin in cattle farms of Mexico. Revista Brasileira de Parasitologia Veterinaria. 23(2): 113-122. https://doi.org/10.1590/s1984-29612014044

Sossai S., Peconick A. P., Sales-Junior P. A., Marcelino F. C., Vargas M. I., Neves E. S. \& Patarroyo J. H. 2005. Polymorphism of the Bm86 gene in South American strains of the cattle tick Boophilus microplus. Experimental and Applied Acarology. 37(3-4): 199214. https://doi.org/10.1007/s10493-005-3262-7

Tellam R. L., Smith D., Kemp D. H. \& Willadsen P. 1992. Vaccinations against ticks. In: Animal Parasite Control Utilizing Biotechnology, (W.K. Yong Ed.). CRC Press. Florida. pp. 303-331. 